\title{
Quality of life in Bipolar affective disorder: Relationship with demographic and clinical variables
}

\author{
Malini Govinadan', Fiaz Ahmed Sattar ${ }^{2}$, Kiran Kumar $\mathbf{K}^{3 *}$, Swapna B ${ }^{4}$, Venugopal VP \\ ${ }^{1}$ Consultant Psychiatrist, ${ }^{2}$ Professor \& Head, ${ }^{3,4}$ Associate Professor, ${ }^{\mathbf{5}}$ Junior Consultant, ${ }^{2-4}$ Dept. of Psychiatry, ${ }^{\mathbf{1} D i s t r i c t ~ H o s p i t a l, ~ T u m k u r, ~}$ \\ Karnataka, ${ }^{2,3}$ Vydehi Institute of Medical Sciences \& Research Centre, Bangalore, Karnataka, ${ }^{4}$ Oxford Institute of Medical Sciences \& \\ Research Centre, Bangalore, Karnataka, ${ }^{5}$ Government Mental Health Centre, Thrissur, Kerala, India
}

\section{*Corresponding Author: Kiran Kumar K}

Email: drkiran.psychiatry@gmail.com

\begin{abstract}
Background and Objectives: Bipolar Affective Disorder (BPAD) is a complex, episodic and heterogeneous condition leading to impairment of Quality of life (Qol). The current study was undertaken with the aim to assess the Quality of Life of patients with Bipolar Affective Disorder, compare it with general population and to examine the correlation of socio-demographic and clinical variables with the Quality of Life.

Methodology: This was a cross-sectional case-control study. Hundred consecutive patients diagnosed to have BPAD as per ICD-10 currently euthymic who fulfilled the inclusion and exclusion criteria and gave informed consent were included for the study. Fifty healthy controls were selected from the general population after clinical interview. Subjects were administered YMRS and HAM-D followed by assessment of QoL using WHOQOL-BREF (World Health Organization Quality of Life -Brief version). The data was analysed using SPSS package version 15.0.

Results: Comparison of the four domain scores of WHOQOL-BREF showed that BPAD patients Qol was significantly impaired than the healthy controls (Physical health, p<0.0001; Psychological, p<0.0001; Social relationships, $\mathrm{p}<0.0003$ and Environmental, p<0.0004). In comparison to healthy controls, the overall perception of QoL (Q1) and overall perception of health (Q2) was significantly lower in the BPAD patients $(\mathrm{Q} 1, \mathrm{p}=0.003$ and $\mathrm{Q} 2, \mathrm{p}<0.001)$. Socio-demographic variables like marital status, type of family, educational status and place of residence had a significant impact on the QoL. Among the clinical variables assessed, early age of onset, number of depressive episodes, HAM-D scores, number of previous hospitalizations and suicidal attempts had a significant negative correlation with QoL. The time elapsed since the last episode had a significant positive correlation with the QoL indicating that longer euthymic period was associated with better QoL.

Conclusion: The emerging body of research has shown that the BPAD has a negative effect on the QoL even during periods of euthymia and remission. A number of socio-demographic and clinical variables have a bearing on the QoL of patients with BPAD.
\end{abstract}

Keywords: Bipolar affective disorder, Quality of life, Remission.

\section{Introduction}

The term Quality of Life (QoL) has become an important construct for all clinicians who are concerned about measuring functional recovery of patients. QoL is a broad ranging concept affected in a complex way by the person's physical health, psychological state, personal beliefs, social relationships and their relationship to salient features of their environment. According to the definition proposed by WHO "Quality of Life is an individual's perception of their position in life in the context of the culture and value systems in which they live and in relation to their goals, expectations, standards and concerns". ${ }^{1}$ It has been suggested that psychiatric disorders are associated with greater impairment in QoL than medical disorders and that there is a distinct pattern of impairment associated with various mental disorders.

The episodic nature of Bipolar Affective Disorder (BPAD) with its many remissions and symptom rich periods of exacerbation, can affect an individual's physical, emotional, social and functional well-being and significantly impact their overall quality of life. ${ }^{2}$ A person suffering from BPAD, apart from facing the symptoms and complications of the illness and the resulting psychosocial impairments also has to contend with stigma and discrimination due to the illness. Due to these reasons many clinicians are increasingly concerned about the QoL of patients with BPAD beyond mere abatement of symptoms. BPAD is responsible for the loss of more disability-adjusted life years than all forms of cancer or major neurologic conditions such as epilepsy and Alzheimer disease, primarily because of its early onset and chronicity across the life span. ${ }^{3}$

A gamut of demographic and clinical variables can influence QoL in patients with BPAD. Gutie'rrez-Rojas L et al examined quality of life in BPAD and found that increasing age was significantly associated with lower QoL. ${ }^{4}$ Sierra $\mathrm{P}$ et al analysed demographic variables of patients with BPAD and found no difference in QoL with respect to marital status, sex, or employment situation. ${ }^{2}$ Earlier studies found women scoring lower on the qualityof-life subscales. ${ }^{4,5}$ While Kebede D et al demonstrated that male sex, rural residence and being married were associated with better functional outcome. ${ }^{6}$ Research has shown that patients with bipolar disorder experience loss of productivity, loss of income due to few paid working hours and unemployment due to morbidity. ${ }^{7,8}$ Romans and McPherson found that BPAD patients have impoverished social relationships and increased rates of marital failure. ${ }^{9}$ All these demographic factors greatly impact the QoL of patients with BPAD. 
Several clinical variables have been associated with poor functional outcome, such as comorbid substance abuse, the side-effects of medication, prior history of psychotic symptoms, low premorbid functioning, persistent subsyndromal fluctuations, the number of prior episodes, prior admissions, younger age of onset and persistent cognitive dysfunction. ${ }^{[10]}$ Another study found low QoL was associated with the length of illness, the presence of depressive symptoms, nicotine dependence and the lack of social support. ${ }^{4}$

Quality of life studies in BPAD patients have so far documented the areas of reduced life satisfaction and functioning, but few have studied the factors that affect QoL. Thus, assessment of QoL in BPAD patients and the study of the predictors of the QoL will help to enrich the understanding of patient's progress and guide the management regimens to achieve complete wellbeing. Therefore, this study was undertaken with the aim to assess the Quality of Life of patients with BPAD, compare it with general population and to examine the correlation of sociodemographic and clinical variables with the Quality of Life.

\section{Methodology}

This was a cross-sectional case-control hospital-based descriptive study. Ethical clearance was obtained from the Institutional ethics committee. One hundred consecutive patients diagnosed as having BPAD as per International Classification of Diseases-10 (ICD-10) criteria in remission and in the age group of 18-65 years of either sex were included in the study after obtaining informed consent and applying inclusion and exclusion criteria. For the purpose of the study, remission was defined as absence or minimal symptoms of both mania and depression for at least 1 week. (As defined in study by Hirschfeld RM et al. ${ }^{11}$ This operational definition was measured as YMRS and HAM-D scores of $\leq 7$. Fifty healthy controls were selected from the general population after a clinical interview. The cases and controls included in the study were interviewed and the socio-demographic variables were recorded in the semistructured proforma designed for the study. Patients were first administered the Young Mania Rating Scale (YMRS) followed by Hamilton Depression Rating Scale (HAM-D 17) and Quality of Life was assessed using World Health Organisation Quality of Life Assessment-Brief (WHOQOLBREF) which was administered in the end of the interview. All the instruments were administered in a single session of one to one and half hour's duration.

\section{Analysis of data}

Data was tabulated and coded. Data was analysed using SPSS package version 15.0. The data was expressed using mean, median and standard deviation for continuous variables and frequency and percentages for categorical variables. Comparison of mean values between groups was done using Independent Sample t test. Relationship between continuous variables was studied using Pearson's correlation coefficient. Association between categorical variables was established by Chi square test/Fishers t test and $\mathrm{p}<0.05$ was considered as significant and $\mathrm{p}<0.005$ was considered as highly significant.

\section{Results}

Majority of the study population were aged between 18 to 48 years $(87 \%)$, most of them were from the rural areas (78\%) and $75 \%$ of the patients were married and most of them were educated up to 10th standard (62\%). Patients mainly hailed from joint families $(67 \%)$ and belonged to class III (54\%) and class IV (35\%) socio-economic status (Modified B.G. Prasad classification). There was no significant difference between the age, gender distribution, marital status and socio-economic status between the cases and controls. However, there was a statistically significant difference in the education levels, occupational status and place of residence between the two groups. It was found that the controls had better educational qualification, majority of them were employed and hailed from urban areas and lived in nuclear families. (Table 1)

In comparison to healthy controls, the overall perception of QoL (Q1) and overall perception of health (Q2) was significantly lower in the BPAD patients $(\mathrm{Q} 1, \mathrm{p}$ $=0.003$ and $\mathrm{Q} 2, \mathrm{p}<0.001)$. (Table $2 \& 3$ ) Comparison of the four domain scores of WHOQOL-BREF showed that BPAD patients QoL was significantly impaired than the healthy controls (Physical health, $\mathrm{p}<0.0001$; Psychological, $\mathrm{p}<0.0001$; Social relationships, $\mathrm{p}<0.0003$ and Environmental, $\mathrm{p}<0.0004)$. (Table 4)

On analysis of the impact of socio-demographic factors on the QoL we found that marital status, type of family, educational status and place of residence had a significant impact on the QoL. It was found that being married had a positive impact on QoL in the psychological and social relationships domain of WHOQOL-BREF. Living in a joint family led to a significantly higher scores in the social relations and environmental domains. Higher educational status had a significant positive correlation with QoL in the social relationship domain. Rural residence had a negative impact on the psychological and social relationships domain of the WHOQOL-BREF. Other socio-demographic variables such as age, gender and occupational status did not have a significant effect on QoL. (Table 5 \& 6)

Among the clinical variables assessed, number of depressive episodes had a highly significant correlation with physical health, psychological and environmental domain of the QoL indicating that number of past depressive episodes was a strong determinant of QoL. It was found that early onset of illness (before the age of 18years) was associated with more impaired QoL in the psychological domain. Number of hospitalizations had a highly significant statistical relationship to the psychological domain indicating that a greater number of hospital admissions had a negative impact on the QoL. Suicide attempts was found to have a significant correlation to the physical health, psychological and social relationships domain of QoL. The study found that longer duration of time elapsed since the last episode led to better QoL in the domains of physical health, psychological and social relationships. (Table $7 \& 8$ ) 
Table 1: Socio-demographic Profile (N=150)

\begin{tabular}{|c|c|c|c|}
\hline $\begin{array}{c}\text { Socio-demographic } \\
\text { Variable }\end{array}$ & $\begin{array}{c}\text { Cases }(\mathbf{n \%}) \\
(\mathbf{n}=100)\end{array}$ & $\begin{array}{c}\text { Controls (n\%) } \\
(\mathbf{n}=\mathbf{5 0})\end{array}$ & $\begin{array}{c}\text { p Value } \\
\text { (Test) }\end{array}$ \\
\hline \multicolumn{4}{|l|}{ Age group } \\
\hline $18-28$ & $39(39)$ & $17(34)$ & \multirow{7}{*}{$\begin{array}{c}\mathrm{p}=0.496 \\
\text { (Independent Samples Test) }\end{array}$} \\
\hline $29-38$ & $26(26)$ & $13(26)$ & \\
\hline $39-48$ & $22(22)$ & $8(16)$ & \\
\hline $49-58$ & $9(9)$ & $12(24)$ & \\
\hline $58-65$ & $4(4)$ & $0(0)$ & \\
\hline Total & $100(100)$ & $50(100)$ & \\
\hline MEAN AGE \pm SD & $34.89 \pm 11.0$ & $36.26 \pm 11.0$ & \\
\hline \multicolumn{4}{|l|}{ Gender } \\
\hline Male & $54(54)$ & $26(52)$ & \multirow{3}{*}{$\begin{array}{c}\mathrm{p}=0.863 \\
\text { (Fisher's Exact Test) }\end{array}$} \\
\hline Female & $46(46)$ & $24(48)$ & \\
\hline Total & 100 & $50(100)$ & \\
\hline \multicolumn{4}{|l|}{ Marital Status } \\
\hline Married & $75(75)$ & $32(64)$ & \multirow{4}{*}{$\begin{array}{c}\mathrm{p}=0.249 \\
(\text { Chi-Square Test) }\end{array}$} \\
\hline Unmarried & $24(24)$ & $18(36)$ & \\
\hline Divorced & $1(1)$ & $0(0)$ & \\
\hline Total & $100(100)$ & $50(100)$ & \\
\hline \multicolumn{4}{|l|}{ Educational Status } \\
\hline Illiterate & $7(7)$ & $7(14)$ & \multirow{5}{*}{$\begin{array}{c}\mathbf{p}=\mathbf{0 . 0 0 7} * \\
(\text { Chi-Square Test })\end{array}$} \\
\hline Primary school & $9(9)$ & $8(16)$ & \\
\hline Up to $10^{\text {th }}$ std & $62(62)$ & $16(32)$ & \\
\hline Degree & $22(22)$ & $19(38)$ & \\
\hline Total & 100 & $50(100)$ & \\
\hline \multicolumn{4}{|l|}{ Family Type } \\
\hline Joint & $67(67)$ & $24(48)$ & \multirow{3}{*}{$\begin{array}{c}\mathrm{p}=0.055 \\
\text { (Fisher's Exact Test) }\end{array}$} \\
\hline Nuclear & $33(33)$ & $26(52)$ & \\
\hline Total & $100(100)$ & $50(100)$ & \\
\hline \multicolumn{4}{|l|}{ Occupation } \\
\hline Student & $9(9)$ & $3(6)$ & \multirow{7}{*}{$\begin{array}{c}\mathbf{p}=\mathbf{0 . 0 0 1} * * \\
(\text { Chi-Square Test })\end{array}$} \\
\hline Unskilled & $5(5)$ & $5(10)$ & \\
\hline Semiskilled & $8(8)$ & $15(30)$ & \\
\hline Skilled & $8(8)$ & $9(18)$ & \\
\hline Business & $20(20)$ & $4(8)$ & \\
\hline Unemployed & $50(50)$ & $14(28)$ & \\
\hline Total & $100(100)$ & $50(100)$ & \\
\hline \multicolumn{4}{|l|}{ Residence } \\
\hline Rural & $78(78)$ & $23(46)$ & \multirow{3}{*}{$\begin{array}{c}\mathbf{p}<\mathbf{0 . 0 0 1} * * \\
\text { (Fisher's Exact Test) }\end{array}$} \\
\hline Urban & $22(22)$ & $27(54)$ & \\
\hline Total & $100(100)$ & $50(100)$ & \\
\hline \multicolumn{4}{|l|}{ SES } \\
\hline Class I & $2(2)$ & $1(2)$ & \multirow{6}{*}{$\begin{array}{c}\mathrm{p}=0.759 \\
\text { (Chi-Square Test) }\end{array}$} \\
\hline Class II & $6(6)$ & $6(12)$ & \\
\hline Class III & $54(54)$ & $21(42)$ & \\
\hline Class IV & $35(35)$ & $15(30)$ & \\
\hline Class V & $3(3)$ & $2(4)$ & \\
\hline Total & $100(100)$ & $50(100)$ & \\
\hline
\end{tabular}


Table 2: Overall perception of Quality of Life (N=150) (Q-1)

\begin{tabular}{|c|c|c|c|}
\hline Q-1 Scores & $\begin{array}{c}\text { Cases } \\
(\mathbf{n = 1 0 0})\end{array}$ & $\begin{array}{c}\text { Controls } \\
(\mathbf{n = 5 0})\end{array}$ & $\begin{array}{c}\text { p Value } \\
\text { (Test) }\end{array}$ \\
\hline Very Poor (1) & $5(5 \%)$ & $0(0 \%)$ & \\
\hline Poor (2) & $9(9 \%)$ & $0(0 \%)$ & \multirow{2}{*}{$\begin{array}{c}\text { 0.003** } \\
\text { (Chi-Square Test) }\end{array}$} \\
\hline Neither Poor nor Good (3) & $47(47 \%)$ & $19(38 \%)$ \\
\hline Good (4) & $39(39 \%)$ & $28(56 \%)$ & \\
\hline Very Good (5) & $0(0 \%)$ & $3(6 \%)$ & \\
\hline Mean score ( \pm SD) & $3.20( \pm 0.794)$ & $3.68( \pm 0.581)$ & \\
\hline
\end{tabular}

Table 3: Overall perception of health $(\mathrm{N}=150)(\mathrm{Q}-2)$

\begin{tabular}{|c|c|c|c|}
\hline Q-2 Scores & $\begin{array}{c}\text { Cases } \\
(n=100)\end{array}$ & $\begin{array}{c}\text { Controls } \\
(\mathbf{n}=\mathbf{5 0})\end{array}$ & $\begin{array}{c}\text { p Value } \\
\text { (Test) }\end{array}$ \\
\hline Very Dissatisfied (1) & $0(0 \%)$ & $0(0 \%)$ & \multirow{6}{*}{$\begin{array}{c}<\mathbf{0 . 0 0 1} * * \\
\text { (Chi-Square Test) }\end{array}$} \\
\hline Dissatisfied (2) & $21(21 \%)$ & $1(2 \%)$ & \\
\hline Neither satisfied nor dissatisfied (3) & $33(33 \%)$ & $9(18 \%)$ & \\
\hline Satisfied (4) & $46(46 \%)$ & $36(72 \%)$ & \\
\hline Very satisfied (5) & $0(0 \%)$ & $4(8 \%)$ & \\
\hline Mean score $( \pm \mathrm{SD})$ & $3.25( \pm 0.782)$ & $3.86( \pm 0.571)$ & \\
\hline
\end{tabular}

Table 4: Comparison of the four whoqol domains $(\mathrm{N}=150)$

\begin{tabular}{|c|c|c|c|c|c|}
\hline Domains & $\begin{array}{c}\text { Mean Scores } \mathbf{S S D} \\
\text { Cases (n=100) }\end{array}$ & $\begin{array}{c}\text { Transformed } \\
\text { Scores } \\
\mathbf{( 0 - 1 0 0 )}\end{array}$ & $\begin{array}{c}\text { Mean scores } \pm \text { SD } \\
\text { Controls } \\
(\mathbf{n = 5 0})\end{array}$ & $\begin{array}{c}\text { Transformed } \\
\text { Scores } \\
(\mathbf{0 - 1 0 0})\end{array}$ & p Value \\
\hline $\begin{array}{c}\text { Domain 1 } \\
\text { Physical health }\end{array}$ & $23.17( \pm 4.18)$ & 56 & $29.10( \pm 2.90)$ & 81 & $<\mathbf{0 . 0 0 0 1 * *}$ \\
\hline $\begin{array}{c}\text { Domain 2 } \\
\text { Psychological }\end{array}$ & $19.01( \pm 3.38)$ & 56 & $22.44( \pm 2.24)$ & 69 & $<\mathbf{0 . 0 0 0 1 * *}$ \\
\hline $\begin{array}{c}\text { Domain 3 } \\
\text { Social relations }\end{array}$ & $9.31( \pm 2.23)$ & 50 & $10.50( \pm 1.61)$ & 69 & $<\mathbf{0 . 0 0 0 3}^{* *}$ \\
\hline $\begin{array}{c}\text { Domain 4 } \\
\text { Environmental }\end{array}$ & $26.78( \pm 3.70)$ & 63 & $28.94( \pm 3.29)$ & 69 & $<\mathbf{0 . 0 0 0 4}^{* *}$ \\
\hline
\end{tabular}

Table 5: Comparison of QoL Domains with Demographic variables

\begin{tabular}{|c|c|c|c|c|c|}
\hline \multicolumn{2}{|c|}{$\begin{array}{l}\text { Demographic Variables } \\
(\mathbf{N})\end{array}$} & \multirow{2}{*}{$\begin{array}{c}\text { DOMAIN } 1 \\
\text { Physical } \\
\text { health } \\
\text { Mean Scores } \pm \\
\text { SD } \\
23.17( \pm 4.39)\end{array}$} & \multirow{2}{*}{$\begin{array}{c}\text { DOMAIN 2 } \\
\text { Psychological } \\
\text { Mean Scores } \pm \\
\text { SD } \\
18.67( \pm 3.52)\end{array}$} & \multirow{2}{*}{$\begin{array}{c}\text { DOMAIN 3 } \\
\text { Social } \\
\text { relations } \\
\text { Mean Scores } \\
\pm \text { SD } \\
9.17( \pm 2.47)\end{array}$} & \multirow{2}{*}{$\begin{array}{c}\text { DOMAIN 4 } \\
\text { Environmental } \\
\text { Mean Scores } \pm \\
\text { SD } \\
26.93( \pm 3.80)\end{array}$} \\
\hline \multirow{3}{*}{$\begin{array}{c}\text { Male } \\
(\mathrm{N}=80)\end{array}$} & Cases $(n=54)$ & & & & \\
\hline & Controls $(n=26)$ & $29.62( \pm 2.74)$ & $22.23( \pm 2.48)$ & $10.50( \pm 1.77)$ & $29.77( \pm 3.26)$ \\
\hline & $\mathrm{p}$ value & $<0.001 * *$ & $<0.001 * *$ & $0.016 *$ & $0.002 * *$ \\
\hline \multirow{3}{*}{$\begin{array}{l}\text { Female } \\
(\mathbf{N}=70)\end{array}$} & Cases $(n=46)$ & $23.17( \pm 4.34)$ & $19.41( \pm 3.18)$ & $9.48( \pm 1.92)$ & $26.61( \pm 3.61)$ \\
\hline & Controls $(\mathrm{n}=24)$ & $28.54( \pm 3.02)$ & $22.67( \pm 1.97)$ & $10.50( \pm 1.47)$ & $28.04( \pm 3.21)$ \\
\hline & $\mathrm{p}$ value & $<0.001 * *$ & $<0.001 * *$ & $0.017 *$ & 0.096 \\
\hline \multirow{3}{*}{$\begin{array}{r}\text { Married } \\
(\mathrm{N}=\mathbf{1 0 7})\end{array}$} & Cases $(n=75)$ & $23.44( \pm 4.10)$ & $19.47( \pm 3.27)$ & $9.65( \pm 1.96)$ & $27.19( \pm 3.43)$ \\
\hline & Controls $(\mathrm{n}=32)$ & $28.27( \pm 2.86)$ & $22.38( \pm 2.48)$ & $10.59( \pm 1.43)$ & $28.59( \pm 3.21)$ \\
\hline & $\mathrm{p}$ value & $<0.001 * *$ & $<0.001 * *$ & $0.047 *$ & $0.046 *$ \\
\hline \multirow{3}{*}{$\begin{array}{l}\text { Unmarried } \\
(\mathrm{N}=43)\end{array}$} & Cases $(n=25)$ & $22.79( \pm 3.90)$ & $17.92( \pm 3.14)$ & $8.29( \pm 2.72)$ & $25.71( \pm 4.26)$ \\
\hline & Controls $(\mathrm{n}=18)$ & $29.78( \pm 2.92)$ & $22.56( \pm 1.76)$ & $10.33( \pm 1.94)$ & $29.56( \pm 3.45)$ \\
\hline & $\mathrm{p}$ value & $<0.001 * *$ & $<0.001 * *$ & $0.007 *$ & $0.003 * *$ \\
\hline \multirow[b]{2}{*}{ Unemployed } & Cases $(n=50)$ & $23.02( \pm 4.43)$ & $19.50( \pm 3.17)$ & $9.62( \pm 2.14)$ & $26.62( \pm 3.24)$ \\
\hline & Controls $(\mathrm{n}=14)$ & $28.21( \pm 3.04)$ & $22.29( \pm 1.81)$ & $10.71( \pm 1.63)$ & $28.36( \pm 3.56)$ \\
\hline
\end{tabular}




\begin{tabular}{|c|c|c|c|c|c|}
\hline$(\mathrm{N}=64)$ & $\mathrm{p}$ value & $<0.001 * *$ & $<0.001 * *$ & 0.08 & 0.08 \\
\hline \multirow{3}{*}{$\begin{array}{l}\text { Urban } \\
(\mathrm{N}=49)\end{array}$} & Cases $(n=22)$ & $23.60( \pm 4.21)$ & $19.37( \pm 3.15)$ & $9.63( \pm 2.10)$ & $27.08( \pm 3.36)$ \\
\hline & Controls $(n=27)$ & $27.78( \pm 3.23)$ & $21.65( \pm 2.10)$ & $10.39( \pm 1.50)$ & $27.52( \pm 3.20)$ \\
\hline & $\mathrm{p}$ value & $<0.001 * *$ & $<0.001 * *$ & 0.108 & 0.575 \\
\hline \multirow{3}{*}{$\begin{array}{c}\text { Rural } \\
(\mathrm{N}=101)\end{array}$} & Cases $(n=78)$ & $21.64( \pm 3.78)$ & $17.73( \pm 3.90)$ & $8.18( \pm 2.36)$ & $25.73( \pm 4.63)$ \\
\hline & Controls $(n=23)$ & $30.22( \pm 2.04)$ & $23.11( \pm 2.17)$ & $10.59( \pm 1.73)$ & $30.15( \pm 2.91)$ \\
\hline & $\mathrm{p}$ value & $<0.001 * *$ & $<0.001 * *$ & $<0.001 * *$ & $<0.001 * *$ \\
\hline \multirow{3}{*}{$\begin{array}{l}\text { Nuclear Family } \\
\qquad(N=59)\end{array}$} & Cases $(n=33)$ & $22.06( \pm 4.69)$ & $18.55( \pm 3.78)$ & $8.42( \pm 2.29)$ & $25.55( \pm 3.03)$ \\
\hline & Controls $(\mathrm{n}=26)$ & $30.04( \pm 2.05)$ & $23.21( \pm 2.41)$ & $10.75( \pm 1.67)$ & $29.79( \pm 4.17)$ \\
\hline & $\mathrm{p}$ value & $<0.001 * *$ & $<0.001 * *$ & $<0.001 * *$ & $<0.001 * *$ \\
\hline \multirow{3}{*}{$\begin{array}{c}\text { Joint Family } \\
\qquad(\mathrm{N}=91)\end{array}$} & Cases $(n=67)$ & $23.72( \pm 3.82)$ & $19.24( \pm 3.16)$ & $9.75( \pm 2.08)$ & $27.39( \pm 3.30)$ \\
\hline & Controls $(\mathrm{n}=24)$ & $28.23( \pm 3.31)$ & $21.73( \pm 2.12)$ & $10.27( \pm 1.56)$ & $28.15( \pm 3.39)$ \\
\hline & $p$ value & $<0.001 * *$ & $<0.001 * *$ & 0.250 & 0.322 \\
\hline
\end{tabular}

$(\mathrm{N}=150)$

Table 6: Correlation of Socio-demographic profile of Cases with QoL Domains (N=100) (Pearson Correlation)

\begin{tabular}{|c|c|c|c|c|}
\hline Demographic variables & $\begin{array}{c}\text { Domain 1 } \\
\text { (Physical health) }\end{array}$ & $\begin{array}{c}\text { Domain 2 } \\
\text { (Psychological) }\end{array}$ & $\begin{array}{c}\text { Domain 3 } \\
\text { (Social relationships) } \\
\end{array}$ & $\begin{array}{c}\text { Domain } 4 \\
\text { (Environmental) }\end{array}$ \\
\hline Age & $\begin{array}{l}r=0.140 \\
p=0.166\end{array}$ & $\begin{array}{l}r=0.022 \\
p=0.705\end{array}$ & $\begin{array}{l}r=0.121 \\
p=0.179\end{array}$ & $\begin{array}{l}r=0.019 \\
p=0.134\end{array}$ \\
\hline Gender & $\begin{array}{l}r=0.001 \\
p=0.993\end{array}$ & $\begin{array}{l}\mathrm{r}=0.111 \\
\mathrm{p}=0.273\end{array}$ & $\begin{array}{l}\mathrm{r}=0.070 \\
\mathrm{p}=0.489\end{array}$ & $\begin{array}{l}r=0.043 \\
p=0.671\end{array}$ \\
\hline Occupation & $\begin{array}{l}\mathrm{r}=0.036 \\
\mathrm{p}=0.722\end{array}$ & $\begin{array}{l}\mathrm{r}=0.146 \\
\mathrm{p}=0.148\end{array}$ & $\begin{array}{l}\mathrm{r}=0.140 \\
\mathrm{p}=0.166\end{array}$ & $\begin{array}{l}\mathrm{r}=0.043 \\
\mathrm{p}=0.668\end{array}$ \\
\hline Marital status & $\begin{array}{l}r=0.164 \\
p=0.103\end{array}$ & $\begin{array}{c}r=0.273 \\
\mathbf{p}=\mathbf{0 . 0 0 8} *\end{array}$ & $\begin{array}{c}r=0.140 \\
\mathbf{p}=\mathbf{0 . 0 3 7} *\end{array}$ & $\begin{array}{c}r=0.208 \\
\mathbf{p}=\mathbf{0 . 0 4 2} *\end{array}$ \\
\hline Residence & $\begin{array}{l}\mathrm{r}=0.196 \\
\mathrm{p}=0.051\end{array}$ & $\begin{array}{c}r=0.203 \\
\mathbf{p}=\mathbf{0 . 0 4 3}\end{array}$ & $\begin{array}{c}\mathrm{r}=0.270 \\
\mathbf{p}=\mathbf{0 . 0 0 7}\end{array}$ & $\begin{array}{l}\mathrm{r}=0.152 \\
\mathrm{p}=0.131\end{array}$ \\
\hline Family type & $\begin{array}{l}\mathrm{r}=0.187 \\
\mathrm{p}=0.062\end{array}$ & $\begin{array}{l}\mathrm{r}=0.097 \\
\mathrm{p}=0.842\end{array}$ & $\begin{array}{c}r=0.280 \\
\mathbf{p}=\mathbf{0 . 0 0 5} *\end{array}$ & $\begin{array}{c}r=0.235 \\
\mathbf{p}=\mathbf{0 . 0 1 8}\end{array}$ \\
\hline Education & $\begin{array}{l}\mathrm{r}=0.121 \\
\mathrm{p}=0.229\end{array}$ & $\begin{array}{l}r=0.027 \\
p=0.78\end{array}$ & $\begin{array}{l}\mathrm{r}=0.107 \\
\mathrm{p}=0.290\end{array}$ & $\begin{array}{c}r=0.227 \\
\mathbf{p}=\mathbf{0 . 0 2 3} *\end{array}$ \\
\hline
\end{tabular}

Table 7: Correlation of clinical variables with QoL Domains ( $\mathrm{N}=100)$ (Pearson Correlation)

\begin{tabular}{|c|c|c|c|c|}
\hline Clinical variables & $\begin{array}{c}\text { Domain 1 } \\
\text { (Physical health) }\end{array}$ & $\begin{array}{c}\text { Domain 2 } \\
\text { (Psychological) }\end{array}$ & $\begin{array}{c}\text { Domain } 3 \\
\text { (Social relationships) }\end{array}$ & $\begin{array}{c}\text { Domain } 4 \\
\text { (Environmental) }\end{array}$ \\
\hline Duration of illness & $\begin{array}{l}r=-0.148 \\
p=0.141\end{array}$ & $\begin{array}{l}\mathrm{r}=-0.157 \\
\mathrm{p}=0.118\end{array}$ & $\begin{array}{l}\mathrm{r}=-0.043 \\
\mathrm{p}=0.674\end{array}$ & $\begin{array}{c}\mathrm{r}=-0.157 \\
\mathrm{p}=0.119\end{array}$ \\
\hline $\begin{array}{c}\text { Time elapsed since last } \\
\text { episode }\end{array}$ & $\begin{array}{c}\mathrm{r}=0.30 \\
\mathbf{p}=\mathbf{0 . 0 0 2} * *\end{array}$ & $\begin{array}{c}r=0.202 \\
\mathbf{p}=\mathbf{0 . 0 4 4} *\end{array}$ & $\begin{array}{c}\mathrm{r}=0.388 \\
\mathbf{p}=\mathbf{0 . 0 0 1} * *\end{array}$ & $\begin{array}{l}\mathrm{r}=0.250 \\
\mathrm{p}=0.012\end{array}$ \\
\hline $\begin{array}{l}\text { No. of depressive } \\
\text { episodes }\end{array}$ & $\begin{array}{c}\mathrm{r}=-0.311 \\
\mathbf{p}=\mathbf{0 . 0 0 2} * *\end{array}$ & $\begin{array}{c}r=-0.376 \\
\mathbf{P}<0.001 * *\end{array}$ & $\begin{array}{l}r=-0.75 \\
p=0.457\end{array}$ & $\begin{array}{c}\mathrm{r}=-0.355 \\
\mathrm{p}=\mathbf{0 . 0 0 1} * *\end{array}$ \\
\hline No. of manic episodes & $\begin{array}{l}r=-0.146 \\
p=0.147\end{array}$ & $\begin{array}{l}\mathrm{r}=-0.121 \\
\mathrm{p}=0.231\end{array}$ & $\begin{array}{c}\mathrm{r}=-0.143 \\
\mathrm{p}=0.155\end{array}$ & $\begin{array}{l}\mathrm{r}=-0.014 \\
\mathrm{p}=0.883\end{array}$ \\
\hline No. of mixed episodes & $\begin{array}{c}\mathrm{r}=0.15 \\
\mathrm{p}=0.884\end{array}$ & $\begin{array}{l}\mathrm{r}=0.143 \\
\mathrm{p}=0.155\end{array}$ & $\begin{array}{l}\mathrm{r}=0.214 \\
\mathrm{p}=0.052\end{array}$ & $\begin{array}{l}\mathrm{r}=-0.082 \\
\mathrm{p}=0.419\end{array}$ \\
\hline $\begin{array}{c}\text { No. of hypomanic } \\
\text { episode }\end{array}$ & $\begin{array}{c}r=-0.135 \\
p=0.180\end{array}$ & $\begin{array}{l}\mathrm{r}=0.029 \\
\mathrm{p}=0.774\end{array}$ & $\begin{array}{l}\mathrm{r}=-0.57 \\
\mathrm{p}=0.575\end{array}$ & $\begin{array}{l}\mathrm{r}=-0.103 \\
\mathrm{p}=0.308\end{array}$ \\
\hline No. of admissions & $\begin{array}{l}\mathrm{r}=-0.232 \\
\mathbf{p}=\mathbf{0 . 0 2 0} *\end{array}$ & $\begin{array}{c}\mathrm{r}=-0.283 \\
\mathbf{p}=\mathbf{0 . 0 0 4} * *\end{array}$ & $\begin{array}{l}\mathrm{r}=-0.99 \\
\mathrm{p}=0.329\end{array}$ & $\begin{array}{l}\mathrm{r}=-0.244 \\
\mathrm{p}=\mathbf{0 . 0 2 5} *\end{array}$ \\
\hline
\end{tabular}


Table 8: Comparison of QoL Domains with cinical variables (N=100)

\begin{tabular}{|c|c|c|c|c|c|}
\hline & cal Variables & \multirow{2}{*}{$\begin{array}{c}\text { Domain 1 } \\
\text { Physical } \\
\text { health } \\
\text { Mean Scores } \pm \\
\text { SD } \\
22.27( \pm 3.08)\end{array}$} & \multirow{2}{*}{$\begin{array}{c}\text { Domain 2 } \\
\text { Psychological } \\
\text { Mean Scores } \pm \\
\text { SD } \\
18.10( \pm 2.63)\end{array}$} & \multirow{2}{*}{$\begin{array}{c}\text { Domain 3 } \\
\text { Social } \\
\text { relations } \\
\text { Mean Scores } \\
\pm \text { SD } \\
8.80( \pm 2.31)\end{array}$} & \multirow{2}{*}{$\begin{array}{c}\text { Domain 4 } \\
\text { Environmental } \\
\text { Mean Scores } \pm \\
\text { SD } \\
27.37( \pm 3.67)\end{array}$} \\
\hline \multirow{3}{*}{$\begin{array}{l}\text { Age at onset of } \\
\text { BPAD }\end{array}$} & Early Onset $(n=30)$ & & & & \\
\hline & Late Onset $(n=70)$ & $23.56( \pm 4.53)$ & $19.40( \pm 3.60)$ & $9.53( \pm 2.17)$ & $25.40( \pm 3.43)$ \\
\hline & $\mathrm{p}$ value & 0.103 & $0.047 *$ & 0.136 & 0.014* \\
\hline \multirow{3}{*}{$\begin{array}{c}\text { Past Psychotic } \\
\text { Episode }\end{array}$} & Psychosis Present $(\mathrm{n}=32)$ & $22.61( \pm 3.92)$ & $18.27( \pm 3.74)$ & $8.88( \pm 2.28)$ & $25.88( \pm 4.35)$ \\
\hline & Psychosis Absent $(n=67)$ & $23.45( \pm 4.30)$ & $19.37( \pm 3.15)$ & $9.52( \pm 2.19)$ & $27.22( \pm 4.35)$ \\
\hline & $p$ value & 0.347 & & 0.176 & 87 \\
\hline \multirow{3}{*}{$\begin{array}{c}\text { Past Suicide } \\
\text { Attempt }\end{array}$} & Suicide Attempt $(\mathrm{n}=19)$ & $20.95( \pm 3.90)$ & $16.53( \pm 3.16)$ & $8.00( \pm 2.53)$ & $25.05( \pm 4.51)$ \\
\hline & No Attempt $(n=81)$ & $23.69( \pm 4.09)$ & $19.59( \pm 3.17)$ & $9.62( \pm 2.05)$ & 27.19 \\
\hline & $\mathrm{p}$ value & 0.009* & & $0.004 * *$ & \\
\hline \multirow{3}{*}{$\begin{array}{c}\text { Drug } \\
\text { Compliance }\end{array}$} & Present $(n=57)$ & $23.54( \pm 4.82)$ & $19.46( \pm 3.70))$ & $9.19( \pm 2.46)$ & $27.46( \pm 3.94)$ \\
\hline & Absent $(n=43)$ & $22.67( \pm 4.13)$ & $18.42( \pm 2.82)$ & $9.47( \pm 1.89)$ & $25.88( \pm 3.17)$ \\
\hline & $\mathrm{p}$ value & 0.278 & 0.129 & 0.549 & 0.035* \\
\hline \multirow{3}{*}{$\begin{array}{c}\text { Family H/O } \\
\text { Psychiatric } \\
\text { Illness }\end{array}$} & Present $(n=21)$ & $22.71( \pm 3.27)$ & $17.71( \pm 3.03)$ & $8.00( \pm 2.30)$ & $25.14( \pm 3.86)$ \\
\hline & Absent $(n=79)$ & $23.29( \pm 4.40)$ & $19.35( \pm 3.40)$ & $9.66( \pm 2.09)$ & $27.22( \pm 3.55)$ \\
\hline & $\mathrm{p}$ value & 0.577 & 0.048* & $0.002 * *$ & 0.022* \\
\hline
\end{tabular}

\section{Discussion}

The Quality of Life of cases with BPAD $(n=100)$ and healthy controls $(\mathrm{n}=50)$ was assessed using the WHOQOLBREF questionnaire which consists of two items from the overall QoL and general health and 24 items of satisfaction that are divided into four domains: Physical health with 7 items, Psychological health with 6 items, Social relationships with 3 items and Environmental health with 8 items.

\section{Overall Perception of QoL: (Table 2)}

The overall perception of QoL was assessed in the WHOQOL-BREF by the 'benchmark' item on overall QoL (Q-1). Our study found that the overall perception of QoL was significantly lower in the cases as compared to that of the healthy controls $(\mathrm{Q}-1, \mathrm{p}=0.003)$. Similar results have been reported by various studies done on QoL in BPAD by Sierra $\mathrm{P}$ et al, ${ }^{2}$ Gutie'rrez-Rojas L et $\mathrm{al}^{4}$ and Arnold $\mathrm{LM}^{12}$ et al. Among the cases, most of them (47\%) reported that they perceived their overall quality of life was neither poor nor good, followed by $39 \%$ of them who perceived quality of life to be good. This perhaps may be the indicator of a positive impact of remission on the quality of their lives. It can also be noted that self-reports of QoL by bipolar patients are likely to be influenced by 'mood bias' or cognitive distortions regarding self-concept and functioning. Studying remitted or euthymic bipolar patients (as in the present study) can reduce this bias. For these patients, the euthymic state offers a chance to reintegrate into society and a healthy lifestyle. The instrument in general uses a cut-off period of past four weeks to assess the overall quality of life and our euthymic patient population benefited from this criterion. However, euthymic patients are not necessarily asymptomatic as many have mild sub-syndromal symptoms, and studies by Michalak et $\mathrm{al}^{13,14}$ have demonstrated that even residual depressive symptoms can be strongly associated with impaired QoL. Not surprisingly in our study about $9 \%$ and $5 \%$ of the patient population reported poor and very poor overall quality of life even in periods of remission. Some limitation arises while comparing "subjective" self-report of patients/controls perception of their overall QoL with an instrument like WHOQOL-BREF. The WHOQOL is based on a purely subjective evaluation, to assess the perceived quality of life, and in this way differs from many other instruments and it approaches the quality of life as a multidimensional concept. So other dimensions apart from the health status also plays an important role in the overall quality of life and this explains why in our study about $38 \%$ of healthy controls rated their overall perception of QoL as neither poor nor good.

\section{Overall perception of health: (Table 3)}

Question 2 of the WHOQOL-BREF scale provides another independent general facet of the subject's overall perception of health. The overall perception of health was significantly lower in the cases as compared to that of the controls $(\mathrm{Q}-2$, $\mathrm{p}<0.001)$. Substantial number of cases rated their overall health as neither satisfied or dissatisfied $(33 \%)$ where as $80 \%$ of the controls were satisfied with their overall health. BPAD is potentially devastating and it encompasses the subsisted experience of responding to symptoms and their accompanying disability, this probably explains the overall dissatisfaction of BPAD patients while perceiving their general health. Measures of QoL, by contrast, are consonant with a bio-psychosocial approach to BPAD, in that they prioritize patient agency, context, meaning-making, and lived experience. Though we measured the QoL of patients in periods of remission the negative effect of the illness was evident. Our study coincides with earlier studies by Sierra P 
et $\mathrm{al}^{2}$ and Kebede et $\mathrm{al}^{6}$ suggesting that bipolar patients experience lower functioning and well-being even in the stable phase of the disorder.

\section{Comparison of the four WHOQOL Domains: (Table 4)}

Means of raw scores were generated for the WHOQOLBREF by organizing the items into facets representing the 4 domains covered by the questionnaire (physical health, psychological, social relationships and environmental). In our study, the QoL of cases with BPAD was significantly lower than in the healthy controls in all the four domains of the WHOQOL-BREF scale. Comparison of the transformed scores of the four domains shows that cases had better scores in the environmental domain ( $\mathrm{t}$ score 63 ) followed by physical health and psychological domains (t score of 56 each) and worse score in social relations (t score 50). These findings correspond to the findings of studies by IsHakk et al, ${ }^{15}$ Sierra P et al, ${ }^{2}$ Gutie'rrez-Rojas L et al ${ }^{4}$ and Arnold LM et $\mathrm{al}^{12}$ who have reported that QoL is to a marked extent lower in patients with BPAD as compared to the general population.

\section{Domain 1 (Physical Health):}

It is not surprising that physical well-being of the BPAD cases was significantly lower than in healthy controls $(p$ $<0.0001$ ) since this domain includes questions related to 7 different components which are physical pain, energy, sleep, ability for Activities of Daily Living (ADL), mobility/activity, dependence on medication and work capacity. Compared to controls, majority of the cases in remission still had problems with physical pain, energy levels and need for treatment. Subsyndromal psychopathology, need for maintenance treatment and reduced energy are all hallmark of BPAD, during various stages of the illness. According to a study by Sofia Brissos et al ${ }^{[16]}$, using the same instrument (WHOQOL-BREF) it was noted that BPAD patients reported worse QoL, especially in the physical and environmental domains.

\section{Domain 2 (Psychological):}

This domain consists of 6 different components which are concentration in work, enjoying life, finding life meaningful, body image, satisfaction with self and negative feelings such as blue mood, anxiety, depression. Lower scores of cases with BPAD on this domain ( $t$ score 56) suggest more extensive problems with self-esteem, body image and cognition. Student $\mathrm{t}$ test indicates highly significant $p$ value $(p<0.0001)$ in this domain between the two groups. Several other studies have compared Health Related QoL in patients with BPAD with that of patients with other psychiatric conditions. For example, the NEMESIS study conducted in the Netherlands compared SF-36 scores in 136 adults with DSM-III-R lifetime BPAD with that observed in a variety of other psychiatric disorders. Participants with BPAD showed significantly more impairment in most SF-36 domains compared with other NEMESIS subjects. For example, in the domain of mental health, participants with BPAD type I experienced significantly lower scores (62.3) than people with other mood (75.2), anxiety (74.0), substance use (80.2) or no psychiatric disorders (85.8). ${ }^{17}$ Other research has compared QoL in patients with schizophrenia with that observed in patients with BPAD. For example, Chand and colleagues in India measured QoL via the Quality of Life Enjoyment and Satisfaction Questionnaire (Q-LES-Q) and the WHOQOLBREF in patients with BPAD who were in remission and stabilized on lithium prophylaxis, patients with schizophrenia and healthy controls. ${ }^{18}$ The bipolar group reported significantly better QoL than the schizophrenia group in general well-being, physical health and psychological health on the WHOQOL-BREF.

\section{Domain 3 (Social Relationships):}

This domain consists of 3 different components which assess the quality of interpersonal relationships other than the family, social support and sexual activity. In this domain, cases with BPAD had the lowest scores ( $t$ score 50). This trend seems consistent with the stigmatization of the disorder in society. Social isolation due to the stigma might generally have a dramatic impact on the social domain. Furthermore, social isolation in BPAD may to some extent be seen both as a source and as a consequence of disability associated with these disorders. A highly significant statistical difference was found on comparison of the scores of cases and controls in this domain $(\mathrm{p}<0.0003)$. Several studies have now assessed QoL in inter-episode patients with BPAD. For example, a Canadian research group generated a series of interrelated reports on QoL in euthymic patients with BPAD. Cooke and colleagues ${ }^{19}$ examined levels of HRQOL using the MOS SF-20, a short version of the SF-36. Analysis of SF-20 scores by type of BPAD showed that patients with BPAD type II reported significantly poorer HRQOL than BPAD type I in the areas of social functioning and mental health. The finding of more dysfunction in social area as compared to other areas is similar to that reported by Kumar et al. ${ }^{20}$ Indian studies have shown poor functioning in social relationship in BPAD patients probably because of greater stigma of mental illnesses in India and the prejudices may be affecting patients' chances of functioning in the social area.

\section{Domain 4 (Environmental):}

It consists of 8 components which are security, physical environment, financial support, accessibility of information, leisure activity, home environment, health care and transport. In our study; cases overall environmental domain score was the best ( $\mathrm{t}$ score 63) when compared to healthy controls (t score 69). It is noteworthy in this context that the WHOQOL-BREF scores within the environmental domain did not seem to adequately discriminate, possibly due to the widespread lack of financial resources and related environmental opportunities in India, similar to the restricted conditions in other developing countries. 
Comparison \& correlation of QoL domains with demographic variables: (Table $5 \& 6$ )

Our study did not find any correlation between age of the patients and the QoL domain scores. Several authors have reported that physical component scores worsened with age and was attributed to medical comorbidities and concomitant substance use. ${ }^{21,22}$ But these results have to be interpreted in the light that majority of the patients in our study, belonged to the age groups of 18 to $38 \mathrm{yrs}$ and elderly patients were poorly represented in the study sample. The study did not find any significant correlation of gender with the QoL domain scores. Similar findings were reported by Sierra $\mathrm{P}$ et $\mathrm{al}^{2}$ who found no significant relationship of gender with QoL in BPAD patients. However, Gutie'rrezRojas L et $\mathrm{al}^{4}$ showed that female sex was significantly associated with worse mental QoL and Kebede et $\mathrm{al}^{6}$ found male sex was associated with better functional outcome. A comparison of the QoL domain scores of male patients with male controls and female patients with female controls was also done. It was found that the QoL scores had a highly significant difference in all the domains for male subjects. Among the females a highly significant difference was found in the QoL scores of the first 3 domains (i.e. physical health, psychological and social relationships). Though female cases scored lower than controls in the domain 4 (environmental domain) a statistically significant difference was not found in the QoL scores, indicating that female subjects maybe less satisfied with financial resources, recreation and leisure, home environment and physical safety which results in reduced satisfaction in these aspects among both the female patients and the controls. Thus in our study though gender did not have statistically significant correlation with the QoL domains, we found that female subjects (cases and controls) had poorer scores in the environmental domain which could partly be explained by the male dominated culture of our country wherein the female population mostly do not have financial independence and have less opportunity for optimum living which reflects in their perception of the QoL. The quality of life of married cases and controls was compared, similarly QoL of unmarried cases and controls were compared. It was found that both married and unmarried cases had significantly poorer quality of life in all the WHOQOL domains as compared to the married and unmarried controls respectively. This demonstrates that both married and unmarried cases have a much lower QoL than that of the general population. However, on corelating the various domains of QoL of married cases with unmarried cases, our study found that there was a statistically significant difference between married and unmarried patients in the psychological $(\mathrm{p}=0.008)$, social relationships $(\mathrm{p}=0.037)$ and environmental domains $(\mathrm{p}=0.042)$ of $\mathrm{QoL}$ indicating that marriage had a positive impact on the QoL. Married patients reported that they felt life is more meaningful and enjoyable, they had lesser negative feelings, were more satisfied with personal relationships and sexual activity and further expressed a sense of safety and security. This finding corresponds to the reports of Kebede D et $\mathrm{al}^{6}$ who found being married is associated with a better QoL. The authors proposed that marriage relationships may signify increased familial and social support enhancing better follow-up and treatment compliance leading to better outcome. However, Gutie'rrez-Rojas $\mathrm{L}$ et $\mathrm{al}^{4}$ found that among female patients those currently married showed worse physical QoL than those non married. The authors interpreted this as an effect of the family burden on the physical health in married women. In our study no significant difference was found in the physical domain scores, this could be because in the WHOQOL-BREF social relations domain had highest content validity whereas physical domain had low content validity. ${ }^{23}$ Our study found a significant correlation between educational status and the environmental domain of QoL $(p=0.023)$ indicating that cases with higher education had a better QoL as compared those with lower educational status but no significant correlation was found in the physical, psychological and social relationships domains. These findings are partly concurrent with the findings of Sofia Brissos et al. who reported that educational level correlated positively with all the WHOQOL-BREF domains, indicating that BPAD patients with higher educational level report a better global QoL. ${ }^{16}$ Low QoL in the environmental domain among the lesser educated cases can be explained as lower educational status may result in reduced opportunities to earn well, financial problems in turn lead to poor housing and lesser opportunities for leisure leading to lower perception of QoL in the environmental domain. Patients with BPAD experienced significantly lower QoL in all the domains as compared to controls when living in a nuclear family $(p=0.000$ ). Among the subjects living in a joint family setting there was a significant difference in the QoL in the physical health and psychological domains of QoL $(\mathrm{p}<0.001)$, but no significant difference in the social relationships $(\mathrm{p}=0.250)$ and environmental domains $(\mathrm{p}=0.322)$. The lack of statistically significant differences in the scores of social and environmental domains between patients and controls may be because, in the WHOQOLBREF used for measuring QoL in our study, the environment domain does not contribute strongly as a component in the QoL measurement. Other validation studies have also shown that the ability of the WHOQOL to distinguish between and across populations is mainly observed in the physical health and psychological health domains rather than in the environment and social relationships domains. ${ }^{[24-26]}$ On corelating the QoL of cases living in nuclear families with that of the cases in joint families, a statistically significant difference was found in the domain 3 (social relations) and domain 4 (environmental) indicating that QoL is better for cases hailing from a joint family as compared to those hailing from a nuclear family ( $\mathrm{p}=0.005$ social relations; $\mathrm{p}=0.018$ environmental domain). This could be because a joint family system is conducive for better social support and patients hailing from joint families reported more satisfaction with personal relationships and social support. Also, joint families may provide the patient a sense of safety and security and perhaps pooled financial resources in the 
joint family system provide the patients with better financial resources, thereby contributing to improvement in the environmental domain of QoL. A comparison of the QoL of unemployed cases with unemployed controls revealed that the scores in the physical health and the psychological domains $(\mathrm{p}<0.001)$ were significantly lower in the patients as compared to controls. However, there was no significant difference in the social and environmental domains in cases and controls. Literature states that unemployment adversely affects life satisfaction, no single aspect of the job is important in itself rather, it is the presence or absence of a job that is crucial. ${ }^{27}$ Thus unemployment adversely effects the QoL irrespective of the health status (of being mentally ill or healthy). This finding is reflected in our study where in unemployed cases had impaired QoL in the social and environmental domains which did not differ significantly from that of the controls. However, there was a statistically significant difference in the QoL in physical and psychological domains of patients when compared to controls indicating that patients may have had impaired energy levels, unsatisfactory sleep, increased dependence on medication and reduced working capacity (which are assessed under the physical domain) and psychological factors like negative feelings, reduced self-esteem and poor learning memory and concentration was more likely to affect the patients' perception of wellbeing as compared to the controls. Though, among the cases, when the QoL of employed and unemployed subjects were corelated, no significant correlation of the employment status with the QoL domain scores was noted. A comparison between cases and controls hailing from the urban areas, revealed that the physical and psychological domain scores were significantly lower for cases but no significant difference was found in the social and environmental domain. Patients hailing from the rural areas had significantly lower scores in all the four QoL domains as compared to the controls. Further on corelating the rural and urban patients with various domains, a statistically significant difference was found in the in QoL scores on the psychological and social relations domain of WHOQOL-BREF $(\mathrm{p}=0.043$ psychological domain; $\mathrm{p}$ $=0.007$ social relationships domain). In the Indian scenario the rural population have to deal with poor infrastructure, less financial resources, poor access to health care and reduced opportunity to recreation and leisure activities leading to impairment of the QoL.

\section{Comparison \& correlation of QoL domains with clinical} variables: (Table $7 \& 8$ )

The study found that cases with early onset (<18years) scored lower in all the domains of QoL. However statistically significant difference was detected only in the psychological domain of QoL ( $\mathrm{p}=0.047)$ and Environmental domains $(p=0.014)$. These findings are similar to the findings of studies conducted by Perlis et al., ${ }^{28}$ Suominen K et $\mathrm{al}^{29}$ and Carlson et $\mathrm{al}^{30}$ who reported that subjects with earlier onset had more lifetime manic, depressive, and mixed phases, suggesting that they have more phases during their mood episodes. Also, the delay in seeking treatment for mood symptoms is longer among early onset BPAD patients. One possible reason for this might be that subjects who had their first mood symptoms at a young age might have got accustomed to these symptoms, regarding them as part of their temperament or personality, not as representing a disorder. Furthermore, children and adolescents with severe mood symptoms are dependent on the adults around them to initiate the referral process. Judith C. Hays ${ }^{31}$ found that stressful life events were more frequent among BPAD subjects with earlier age of onset while patients with later onset had more instrumental and subjective social support. All these factors have a bearing on the QoL of patients with early onset. Our study found that though QoL is lower in patients with early onset as compared to late onset, the most significant impairment was in the psychological domain. This could be probably be because psychological aspects of QoL which involve assessment of positive feelings, selfesteem, body image, thinking and learning are much more effected when the illness begins at a younger age than in cases when the onset occurs in older individuals. No significant correlation was found between the duration of illness and any of the four domains of QoL scores. Our finding that longer duration of the illness is not significantly correlated to the QoL domains, can be explained as patients in the stable phase of the illness may have come to terms with their limitations and may have lowered expectations and aspirations as the years progress. Thus, we can conclude that though early onset is associated with a worse QoL, longer duration of illness may not always result in further impaired QoL. In our study the average number of depressive episodes was $2.10 \pm 1.630$. The study found a highly significant correlation between the number of depressive episodes and domain scores in the physical health, psychological and environmental domains of WHOQOL $\quad(p=0.002$ physical health; $p<0.001$ psychological; $\mathrm{p}=0.001$ environmental domain). This is similar to the findings of Perlis et $a{ }^{28}{ }^{28}$ Singh et al ${ }^{32}$ and Maina $\mathrm{G}$ et $\mathrm{al}^{33}$ who found that past depressive episodes led to poorer quality of life. Research have indicated a preponderance of depressive symptoms over hypomanic or manic symptoms in patients with both bipolar disorder types I and II. Much of the morbidity in BPAD appears to be a consequence of the depressive phase of the disorder. Studies have shown that subsyndromal symptoms of depression is markedly prevalent in inter episode patients with BPAD and can be predictive of significant functional impairment, specifically impairment in work, home functioning roles, as well as impairment in relationship with family and friends. Peter J.J. Goosens et al proposed that symptoms of depression may be the most important cause of reduced QoL for individuals living with BPAD and residual symptoms of depression contribute to decreased QoL. ${ }^{34}$ The study did not find any significant correlation between the number of manic episodes and the QoL domain scores. The shorter duration of the manic episodes and faster recovery from these episodes as compared to the depressive episodes, and the apparent "supranormal" QoL reported in patients with mania or hypomania ${ }^{35}$ may partly explain the failure to find 
a significant impairment of QoL in BPAD patients following manic episodes. The mean number of hospitalizations was $1.28 \pm 0.996$. The number of admissions had a highly significant correlation with the psychological domain $(\mathrm{p}=0.004)$ and significant correlation with the physical health $(\mathrm{p}=0.020)$ and environmental domains of QoL $(\mathrm{p}=0.025)$. Repeated hospitalizations indicate a more severe illness. It affects the activities of daily living, results in greater dependence on medications, causes negative feelings and reduced self-esteem in the patient besides causing additional burden on financial resources of the family. All these factors explain the significant negative correlation of the QoL to the number of hospital admissions. The time elapsed from the last episode varied from 3 months to 5yrs, the mean time elapsed from the last episode was 0.759 years \pm 0.8292 . There was a highly significant correlation between the time elapsed from last episode and the QoL domain scores in the physical health domain, social relationships domain and in the psychological domain $(\mathrm{p}=0.002, \quad \mathrm{p}=0.001, \quad \mathrm{p}=0.044$ respectively). This indicates that the longer duration the patient is in remission the better is the QoL. In the current study presence of psychotic symptoms in the past episodes did not result in a significant difference in the QoL domain scores. This finding corresponds to the findings of MacQueen GM et $\mathrm{al}^{36}$ who concluded that psychotic patients were more symptomatic during the index episode, but did not differ from the non-psychotic patients on ratings of function and wellbeing when euthymic. This could be because our study was a cross sectional study conducted in the euthymic period and the instrument used (WHOQOLBREF) considered a time frame of 4 weeks for the assessment QoL. Therefore, it is possible that a past episode of psychosis may not have significantly influenced the QoL at the time of examination when the patient was symptom free. In our study $19 \%$ of the cases attempted suicide. On comparing the QoL of suicide attempters with the nonattempters there was a highly significant difference in the psychological domain, physical health and social domain $(\mathrm{p}=0.001, \mathrm{p}=0.009$ and $\mathrm{p}=0.004$ respectively). Similar findings have been noted by Leverich et $\mathrm{al}^{37}$ who concluded that most patients with a history of suicide attempts, had a greater personal history of early traumatic stressors and poorer prognosis in the longitudinal course and Perlis et $\mathrm{al}^{28}$ found that suicide attempts had a significant correlation with early onset BPAD which in turn is associated with poor functioning and QoL. Among the patients $21 \%$ had a family history of psychiatric illness. It was found that the QoL scores in psychological, social and the environmental domains were significantly lower among those with positive family history as compared to those patients with no family history of psychiatric illness. This corresponds to the findings reported by Ross J Baldessarini et $\mathrm{al}^{38}$ who showed that positive family history is associated with poor functional outcomes. Niki Antypa et $\mathrm{al}^{39}$ found patients reporting family history of a mood disorder had an earlier age at onset of depression/mania, more phases, rapid cycling and more suicide attempts and further had lower quality of life as compared to patients without family history. Thus, it is possible that presence of family history of psychiatric illness may lead to earlier onset of illness, more severe course, and more hospitalizations besides the added psychosocial impairment of the family having more than one person effected by mental illness. These factors in turn have a negative effect the QoL.

\section{Conclusion}

The emerging body of research has shown that the BPAD has a negative effect on the QoL even during periods of euthymia and remission. A number of socio-demographic and clinical variables have a bearing on the QoL of patients with BPAD. Further, measuring QoL provides additional important information from the patients' point of view and a knowledge of the predictors related to the quality of life, can contribute to the design of future clinical interventions which can favourably influence the QoL of BPAD patients.

\section{Source of Funding}

Nil.

\section{Conflicts of Interest}

Nil.

\section{References}

1. World Health Organization. WHOQOL Measuring QoL. Geneva. 1998 http://www.who.int/mental_health/media/68.pdf (accessed on 18 October 2019).

2. Sierra P, Livianos L, Rojo L. Quality of life for patients with bipolar disorder: relationship with clinical and demographic variables. Bipolar Disord. 2005;7:159-65.

3. Kathleen RM, Robert Jin. Prevalence and Correlates of Bipolar Spectrum Disorder in the World Mental Health Survey Initiative. Arch Gen Psychiatry. 2011;68(3):241-51.

4. Gutie'rrez-Rojas L, Gurpegui M, Ayuso-Mateos JL, Gutie'rrez-Ariza JA, Ruiz-Veguilla M, Jurado D. Quality of life in bipolar disorder patients: a comparison with a general population sample. Bipolar Disord. 2008:10:625-34.

5. Robb JC, Young TL, Cooke RG, Joffe RT. Gender differences in patients with bipolar disorder influence outcome in the medical outcomes survey (SF 20) subscale scores. J Affect Disord. 1998;49:189-93.

6. Kebede D, Alem A, Shibire T, Deyassa N, Negash A et al. Symptomatic and functional outcome of bipolar disorder in Butajira, Ethiopia. J Affect Disord. 2006;90:239-49

7. Lish JD, Dime-Meenan S, Whybrow PC, Price RA, Hirschfeld RM. The National Depressive and Manic-depressive Association (DMDA) survey of bipolar members. $J$ Affect Disord. 1994;31:281-94.

8. Wyatt RJ, Henter I. An economic evaluation of manicdepressive illness-1991. Soc Psychiatry Psychiatric Epidemiol. 1995;30:213-9.

9. Romans $\mathrm{S}, \mathrm{McPherson} \mathrm{H}$. The social networks of bipolar affective disorder patients. J Affect Disord 1992; 25: 221-8.

10. Martinez-Aran A, Vieta E, Torrent C, Sánchez-Moreno J, Goikolea J.M, Salamero M, et al. Functional outcome in bipolar disorder: the role of clinical and cognitive factors. Bipolar Disord. 2007;9:103-13.

11. Hirschfeld RM, Calabrese JR, Frye MA, Lavori PW, Sachs G, Thase ME, Wagner KD. Defining the clinical course of bipolar disorder: response, remission, relapse, recurrence, and roughening. Psychopharmacol Bull. 2007;40(3):7-14. 
12. Arnold LM, Witzeman KA, Swank ML, McElroy SL, Keck PE Jr. Health-related quality of life using the SF-36 in patients with bipolar disorder compared with patients with chronic back pain and the general population. J Affect. Disord. 2000;57:23539.

13. Michalak EE, Yatham LN, Lam RW. Quality of life in bipolar disorder: a review of the literature. Health Qual Life Out. 2005;3:72-117.

14. Michalak EE, Murray G, Young AH, Lam RW. Burden of Bipolar Depression Impact of Disorder and Medications on Quality of Life. CNS Drugs. 2008;22(5):389-406.

15. IsHak WW, Brown K, Aye SS, Kahloon M, Mobaraki S, Hanna R. Health-related quality of life in bipolar disorder. Bipolar Disord. 2012;14:6-18.

16. Brissos S, Dias VV, Carita AI, Martinez-Arán A. Quality of life in bipolar type I disorder and schizophrenia in remission: Clinical and neurocognitive correlates. Psychiatry Res. 2008;160(1):55-62.

17. Ten Have M, Vollebergh W, Bijl R, Nolen WA. Bipolar disorder in the general population in The Netherlands (prevalence, consequences and care utilisation): results from The Netherlands Mental Health Survey and Incidence Study (NEMESIS). J Affect Disord. 2002;68(2-3):203-13.

18. Chand PK, Mattoo SK, Sharan P. Quality of life and its correlates in patients with bipolar disorder stabilized on lithium prophylaxis. Psychiatry Clin Neurosci. 2004;58:311-8.

19. Cooke RG, Robb JC, Young LT, Joffe RT. Well-Being and Functioning in Patients With Bipolar Disorder Assessed Using the MOS 20-ITEM Short Form (SF-20). J Affect Disord.1996;39(2):93-7.

20. Kumar D, Basu D, Kulhara P, Sharan P. Psychosocial correlates of subsyndromal symptoms and functioning of bipolar patients stabilized on prophylactic lithium. Indian J. Psychiatry. 2001;43:199-205.

21. Fenn HH, Bauer MS, Altshuler L, Evans DR, Williford WO, Kilbourne AM et al. Medical comorbidity and health-related quality of life in bipolar disorder across the adult age span. $J$ Affect Disord. 2005;86(1):47-60.

22. Depp CA, Davis CE, Mittal D, Patterson TL, Jeste DV. Health-related quality of life and functioning of middle-aged and elderly adults with bipolar disorder. J Clinic Psychiatry. 2006;67:215-21.

23. Agnihotri K, Awasthi S, Chandra H, Singh U, Thakur S. Validation of WHO QOL-BREF Instrument in Indian Adolescents. Indian J Pediatr. 2010;77(4):381-6.

24. Saxena S, Quinn K, Sharan P, Naresh B, Yuantao-Hao, Power M:Cross-linguistic equivalence of WHOQOL-100: a study from North India. Qual Life Res 2005, 14:891-7.

25. Wesolowski T, Szyber P: Usage of the WHOQOL-100 as a trial of objective estimation of quality of life in end stage renal disease patients treated with renal transplantation. Pol Merkur Lekarski. 2004;17:260-6.

26. Waagsaether K, Hanestad B, Kalfoss M: The quality of life assessment instrument, WHOQOL-100: translation, adjustment and validation - a study of healthy women and women with pelvic joint syndrome. Qual Life Res. 1999;8:555-665.

27. Morselli PL, Elgie R., Cesana BM. GAMIAN-Europe/BEAM analysis of unemployment, family history, treatment satisfaction and impact of the bipolar disorder on life style. Bipolar Disord. 2004;6:487-97.

28. Perlis RH, Miyahara S, Marangell LB, Wisniewski SR, Ostacher M, Del-Bello MP. Long-Term implications of early onset in bipolar disorder: data from the first 1000 participants in the Systematic Treatment Enhancement Program for Bipolar Disorder (STEP-BD). Biol Psychiatry. 2004;55:875-81.

29. Suominen K, Mantere O, Valtonen H, Arvilommi P, Leppa"ma" ki S, Paunio T, Isometsa" E. Early age at onset of bipolar disorder is associated with more severe clinical features but delayed treatment seeking. Bipolar Disord. 2007:9:698705.

30. Carlson GA, Bromet EJ, Driessens C, Mojtabai R, Schwartz JE. Age at Onset, Childhood Psychopathology and 2-Year Outcome in Psychotic Bipolar Disorder. Am J Psychiatry. 2002;159:307-9.

31. Hays JC, Krishnan KRR, George LK, Blazer DG. Age of first onset of bipolar disorder: demographic, family history, and psychosocial correlates. Depress Anxiety.1998;7:76-82.

32. Singh J, Mattoo SK, Sharan P, Basu D. Quality of life and its correlates in patients with dual diagnosis of bipolar affective disorder and substance dependence. Bipolar Disord. 2005:7:187-91.

33. Maina G, Albert U, Bellodi L, Colombo C, Faravelli C, Monteleone $\mathrm{P}$ et al. Health-related quality of life in euthymic bipolar disorder patients: differences between bipolar I and II subtypes. J Clin Psychiatry. 2007;68: 207-12.

34. Peter JJ Goossens, Erwin G, Hartong M, Elise AM, Knoppertvan der Klein, Theo van Achterberg. Self-Reported Psychopathological Symptoms and Quality of Life in Outpatients with Bipolar Disorder. Perspect Psychiatric Care. 2008;44(4):275-84.

35. Zhang H, Wisniewski SR, Bauer MS, Sachs GS, Thase ME. Comparisons of perceived quality of life across clinical states in bipolar disorder: data from the first 2000 Systematic Treatment Enhancement Program for Bipolar Disorder (STEPBD) participants. Compr Psychiatry. 2006;47:161-8.

36. MacQueen GM, Young LT, Robb JC, Cooke RG, Joffe RT. Levels of functioning and well-being in recovered psychotic versus nonpsychotic mania. J Affect Disord. 1997; 46:69-2.

37. Leverich GS, Altshuler LL, Frye MA, Suppes T, Keck PE, McElroy SL et al. Factors associated with suicide attempts in 648 patients with bipolar disorder in the Stanley Foundation Bipolar Network. J Clin Psychiatry. 2003;64(5):506-15.

38. Ross J Baldessarini, Leonardo Tondo. Age at onset versus family history and clinical outcomes in 1,665 international bipolar-I disorder patients. World Psychiatry. 2012;11(1): 406.

39. Antypa N, Serretti A. Family history of a mood disorder indicates a more severe bipolar disorder. J Affect Disord. 2014;156:178-86.

How to cite this article: Govinadan M, Sattar FA, Kumar KK, Swapna B, Venugopal VP. Quality of life in Bipolar affective disorder: Relationship with demographic and clinical variables. Telangana J Psychiatry. 2020;6(1):47-57. 